\title{
Exponential Sums for Seventh Degree Polynomial
}

\author{
Low Chee Wai ${ }^{1^{*}}$, Siti Hasana Sapar ${ }^{2}$ and Mohamat Aidil Mohamat Johari ${ }^{3}$ \\ 1,2,3 Institute for Mathematical Research, Universiti Putra Malaysia, 43400 UPM Serdang, Selangor Darul \\ Ehsan, Malaysia \\ 2,3 Department of Mathematics, Faculty of Science, Universiti Putra Malaysia, 43400 UPM Serdang, \\ Selangor Darul Ehsan, Malaysia
}

Let $f(x, y)$ be a seventh-degree polynomial with two variables in with complete dominant terms. Suppose $p>7$ is a prime, the exponential sums of polynomial $f(x, y)$ is defined by $S\left(f ; p^{\alpha}\right)=$ $\sum_{x, y \bmod p} e^{\frac{2 \pi i f(x, y)}{p^{a}}}$, where the sum is taken over a complete set of residue modulo $p$. In order to get the value of $S\left(f ; p^{\alpha}\right)$, the cardinality $N\left(g, h ; p^{\alpha}\right)$ must be obtained first. In this paper, we discuss the Newton Polyhedron technique in finding the $p$-adic sizes of common zeros of the partial derivative polynomials $f_{x}$ and $f_{y}$ which derive from $f(x, y)$. Then, the estimation of the cardinality and exponential sums of polynomial $f(x, y)$ will be determined accordingly. For $\alpha>1$, the exponential sums of $f(x, y)$ is given by $\left|S\left(f ; p^{\alpha}\right)\right| \leq \min \left\{p^{2 \alpha}, 36 p^{\alpha+1+36 \delta+6 \omega_{0}+12 q}\right\}$ where $\delta, \omega_{0}, q \geq 0$.

Keywords: $p$-adic sizes, Newton polyhedron, cardinality, exponential sums

\section{INTRODUCTION}

In this paper, $Z_{p}$ denotes as the field of $p$-adic integer. $\Omega_{p}$ denotes as the completion of algebraic closure of the field of rational $p$-adic numbers $Q_{p}$. The highest power of $p$ which divides $x$ is denoted by $\operatorname{ord}_{p} x$.

Loxton \& Smith (1982) estimated the cardinality $N\left(f, p^{\alpha}\right)$ by the $p$-adic sizes of common zeros of partial derivative polynomials associated with $f$ in the neighborhood of points in the product space $\Omega_{p}^{n}, n>0$.

Loxton \& Vaughan (1985) studied the estimation of exponential sums by using the number of common zeros of partial derivative polynomials with respect to $x$ modulo $q$.

Mohd. Atan \& Loxton (1986) used the Newton polyhedral method to obtain the $p$-adic sizes of polynomials in $\Omega_{p}[x, y]$ which is an analogue of Newton polygon in Koblitz (1977). They estimated the cardinality for certain lower-degree polynomials $f(x, y)$ over $Z_{p}$.

The estimations with Newton polyhedron technique for lower degree two-variable polynomials are also found in Mohd. Atan (1986), Chan \& Mohd. Atan (1997), Heng \& Mohd. Atan (1999) as well as Sapar \& Mohd. Atan (2002). However, the results for the higher degree polynomials are less complete.

Then, Sapar \& Mohd. Atan (2009) gave the $p$-adic sizes of common zeros of partial derivative polynomials associated with a quintic form for prime $p>5$.

Yap et al. (2011) showed that the $p$-adic sizes of common zeros of partial derivative polynomials associated with a cubic form can be found explicitly on the indicator diagrams by using Newton polyhedron technique.

Sapar et al. (2013) also investigated the estimation of $p$-adic sizes of common zeros of degree nine polynomial.

Aminudin et al. (2014) continued the research of Yap et al. (2011) on a complete cubic form polynomial. They found that the result is different due to different form of the cubic polynomials. This means different form of polynomials will result different $p$-adic sizes although both of them are cubic polynomials.

Next, Sapar et al. (2014) studied the estimation of $p$ adic sizes of an eighth-degree polynomial. Lasaraiya et al. (2016a) and Lasaraiya et al. (2016b) researched on the

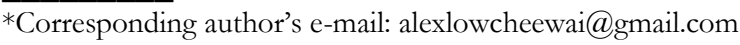


cardinality $N\left(f_{x}, f_{y} ; p^{\alpha}\right)$ of twelfth- and eleventh-degree polynomials respectively.

In this paper, we apply the Newton polyhedron technique to determine the $p$-adic sizes of the partial derivative polynomials of $f(x, y)$ in $Z_{p}[x, y]$ of a degree seven. Then, we obtain the estimation of cardinality and the exponential sums of the polynomial

$$
\begin{gathered}
f(x, y)=a x^{7}+b x^{6} y+c x^{5} y^{2}+d x^{4} y^{3}+e x^{3} y^{4} \\
+k x^{2} y^{5}+m x y^{6}+n y^{7}+r x+s y+t .
\end{gathered}
$$

\section{P-ADIC SIZE OF COMMON ZERO OF POLYNOMIAL}

Sapar \& Mohd. Atan (2002) proved that every point of intersection of the Indicator diagrams, there exist common zeros of both polynomials in $Z_{p}[x, y]$ which $p$-adic sizes correspond to point $\left(\mu_{1}, \mu_{2}\right)$ as in the following.

Theorem 1 Let $\mathrm{p}$ be a prime. Suppose $f$ and $g$ are polynomials in $Z_{p}[x, y]$. Let $\left(\mu_{1}, \mu_{2}\right)$ be a point of intersection of the Indicator diagrams associated with $f$ and $g$ at the vertices or simple points of intersections. Then there are $\xi$ and $\eta$ in $\Omega_{p}^{2}$ satisfying $f(\xi, \eta)=g(\xi, \eta)=0$ and $\operatorname{ord}_{p} \xi=\mu_{1}, \operatorname{ord}_{p} \eta=\mu_{2}$.

The following theorem gives the $p$-adic size of common zero of polynomial that we consider.

Theorem 2 Let $f(x, y)=a x^{7}+b x^{6} y+c x^{5} y^{2}+d x^{4} y^{3}+$ $e x^{3} y^{4}+k x^{2} y^{5}+m x y^{6}+n y^{7}+r x+s y+t$ be a polynomial in $Z_{p}[x, y]$ and $\left(x_{0}, y_{0}\right)$ be a point in $\Omega_{p}^{2}$ with $p>7$ is a prime. Let $\alpha>0$,

$\delta=\max \left\{\operatorname{ord}_{p} a, \operatorname{ord}_{p} b, \operatorname{ord}_{p} c, \operatorname{ord}_{p} d, \operatorname{ord}_{p} e\right.$,

$\left.\operatorname{ord}_{p} k, \operatorname{ord}_{p} m, \operatorname{ord}_{p} n\right\} . \quad$ If $\operatorname{ord}_{p} f_{x}\left(x-x_{0}, y-y_{0}\right)$, $\operatorname{ord}_{p} f_{y}\left(x-x_{0}, y-y_{0}\right) \geq \alpha>\delta$, then there exists $(\xi, \eta)$ such that $f_{x}(\xi, \eta)=0, f_{y}(\xi, \eta)=0$. The $p$-adic sizes are given by

$$
\begin{aligned}
& \operatorname{ord}_{p}\left(\xi-x_{0}\right) \geq \frac{1}{6}(\alpha-34 \delta)-\varepsilon_{1}, \\
& \operatorname{ord}_{p}\left(\eta-y_{0}\right) \geq \frac{1}{6}(\alpha-22 \delta)-\varepsilon_{2}
\end{aligned}
$$

for $\operatorname{ord}_{p}(35 c n-e k)^{2} \neq \operatorname{ord}_{p} 4(21 d n-3 e m)$

$(5 \mathrm{~cm}-\mathrm{kd})$, and

$$
\begin{aligned}
& \operatorname{ord}_{p}\left(\xi-x_{0}\right) \geq \frac{1}{6}(\alpha-34 \delta)-\varepsilon_{3}-\frac{1}{2} \omega_{0}, \\
& \operatorname{ord}_{p}\left(\eta-y_{0}\right) \geq \frac{1}{6}(\alpha-22 \delta)-\varepsilon_{4}-\frac{1}{2} \omega_{0}
\end{aligned}
$$

for $\operatorname{ord}_{p}(35 c n-e k)^{2}=\operatorname{ord}_{p} 4(21 d n-3 e m)$

$(5 \mathrm{~cm}-k d)$, where $\varepsilon_{1}, \varepsilon_{2}, \varepsilon_{3}, \varepsilon_{4}, \omega_{0} \geq 0$.

In order to prove Theorem 2, we take a linear combination of $g=f_{x}(x, y)$ and $h=f_{y}(x, y)$ as $g+\lambda h$.
Then, we do a transformation by letting $U=\left(X+x_{0}\right)+$ $\frac{1}{6} \alpha_{1}\left(Y+y_{0}\right)$ and $V=\left(X+x_{0}\right)+\frac{1}{6} \alpha_{2}\left(Y+y_{0}\right)$ where $\alpha_{1}=$ $\frac{6 b+2 \lambda_{1} c}{6\left(7 a+\lambda_{1} b\right)}$ and $\alpha_{2}=\frac{6 b+2 \lambda_{2} c}{6\left(7 a+\lambda_{2} b\right)}$ that we needed in the following lemma.

Lemma 1 Let $\lambda_{1}, \lambda_{2}$ be the zeros of quadratic function $z(\lambda)$ in the form $z(\lambda)=(21 d n-3 e m) \lambda^{2}+(35 c n-e k) \lambda+$ $(5 \mathrm{~cm}-k d)$. Suppose $p$ is a prime and $a, b, c, d, e, k, m, n$ in $Z_{p}$, then

$\operatorname{ord}_{p}\left(\alpha_{1}-\alpha_{2}\right)=$

$\frac{1}{2} \operatorname{ord}_{p}\left[(35 c n-e k)^{2}-4(21 d n-3 e m)(5 c m-k d)\right]$

$-\operatorname{ord}_{p}(21 d n-3 e m)+\operatorname{ord}_{p}\left(14 a c-6 b^{2}\right)$

$-\operatorname{ord}_{p}\left(7 a+\lambda_{1} b\right)-\operatorname{ord}_{p}\left(7 a+\lambda_{2} b\right)$.

Proof. $\alpha_{1}-\alpha_{2}=\frac{\left(\lambda_{1}-\lambda_{2}\right)\left(14 a c-6 b^{2}\right)}{6\left(7 a+\lambda_{1} b\right)\left(7 a+\lambda_{2} b\right)}$. Take $\operatorname{ord}_{p}$ on both sides and substitute the expression of $\lambda_{1}-\lambda_{2}=$ $\frac{\sqrt{(35 c n-e k)^{2}-4(21 d n-3 e m)(5 c m-k d)}}{(21 d n-3 e m)}$, we obtain: $\operatorname{ord}_{p}\left(\alpha_{1}-\alpha_{2}\right)$

$$
\begin{gathered}
=\frac{1}{2} \operatorname{ord}_{p}\left[(35 c n-e k)^{2}-4(21 d n-3 e m)\right. \\
(5 c m-k d)]-\operatorname{ord}_{p}(21 d n-3 e m) \\
+\operatorname{ord}_{p}\left(14 a c-6 b^{2}\right)-\operatorname{ord}_{p}\left(7 a+\lambda_{1} b\right) \\
-\operatorname{ord}_{p}\left(7 a+\lambda_{2} b\right) .
\end{gathered}
$$

Lemma 2 Let $p>7$ be a prime and $a, b, c, d, e$,

$k, m, n, r, s$ in $Z_{p}$. Suppose $\left(X+x_{0}, Y+y_{0}\right)$ in $\Omega_{p}^{2}, \delta=$ $\max _{\operatorname{ord}} a, \operatorname{ord}_{p} b, \operatorname{ord}_{p} c, \operatorname{ord}_{p} d, \operatorname{ord}_{p} e$,

$\left.\operatorname{ord}_{p} k, \operatorname{ord}_{p} m, \operatorname{ord}_{p} n\right\}$ and $\operatorname{ord}_{p} r, \operatorname{ord}_{p} s \geq \alpha>\delta$.

If $\operatorname{ord}_{p} U=\frac{1}{6} \operatorname{ord}_{p}\left(\frac{r+\lambda_{1} s}{7 a+\lambda_{1} b}\right)$ and $\operatorname{ord}_{p} V=\frac{1}{6} \operatorname{ord}_{p}\left(\frac{r+\lambda_{2} s}{7 a+\lambda_{2} b}\right)$ with the condition $\operatorname{ord}_{p}(35 c n-e k)^{2} \neq \operatorname{ord}_{p} 4(21 d n-$ $3 e m)(5 c m-k d)$ where $=x+\alpha_{1} y \quad$ and $\quad V=x+\alpha_{2} y$, then

$\operatorname{ord}_{p}\left(X+x_{0}\right) \geq \frac{1}{6}(\alpha-34 \delta)$

and

$\operatorname{ord}_{p}\left(Y+y_{0}\right) \geq \frac{1}{6}(\alpha-22 \delta)$.

Proof. Let $x=X+x_{0}$ and $y=Y+y_{0}$. Substitute into $U=$ $x+\alpha_{1} y$ and $V=x+\alpha_{2} y$, we have

$$
\begin{aligned}
& \left(X+x_{0}\right)=\frac{\alpha_{1} V-\alpha_{2} U}{\alpha_{1}-\alpha_{2}}, \\
& \left(Y+y_{0}\right)=\frac{U-V}{\alpha_{1}-\alpha_{2}} .
\end{aligned}
$$

From (2),

$$
\begin{aligned}
\operatorname{ord}_{p}\left(Y+y_{0}\right) \geq & \min \left\{\operatorname{ord}_{p} U, \operatorname{ord}_{p} V\right\} \\
& -\operatorname{ord}_{p}\left(\alpha_{1}-\alpha_{2}\right) .
\end{aligned}
$$

By Lemma 1, we have

$\operatorname{ord}_{p}\left(Y+y_{0}\right)$ 


$$
\begin{aligned}
\geq & \min \left\{\operatorname{ord}_{p} U, \operatorname{ord}_{p} V\right\}-\frac{1}{2} \operatorname{ord}_{p}\left[(35 c n-e k)^{2}\right. \\
& -4(21 d n-3 e m)(5 c m-k d)]+\operatorname{ord}_{p} \\
& (21 d n-3 e m)-\operatorname{ord}_{p}\left(14 a c-6 b^{2}\right)+\operatorname{ord}_{p} \\
& \left(7 a+\lambda_{1} b\right)+\operatorname{ord}_{p}\left(7 a+\lambda_{2} b\right) .
\end{aligned}
$$

Since $\operatorname{ord}_{p}(35 c n-e k)^{2} \neq \operatorname{ord}_{p} 4(21 d n-3 e m)$

$(5 \mathrm{~cm}-k d)$, we consider two cases.

Case (i): $\operatorname{ord}_{p}(35 c n-e k)^{2}>\operatorname{ord}_{p} 4(21 d n-3 e m)(5 c m-$ $k d)$,

Case (ii): $\operatorname{ord}_{p}(35 c n-e k)^{2}<\operatorname{ord}_{p} 4(21 d n-3 e m)(5 \mathrm{~cm}-$ $k d)$.

For Case (i), equation (3) becomes

$$
\begin{aligned}
\operatorname{ord}_{p}\left(Y+y_{0}\right) \geq & \min \left\{\operatorname{ord}_{p} U, \operatorname{ord}_{p} V\right\}-\frac{1}{2} \operatorname{ord}_{p} \\
& (5 c m-k d)+\frac{1}{2} \operatorname{ord}_{p}(21 d n \\
& -3 e m)-\operatorname{ord}_{p}\left(14 a c-6 b^{2}\right) \\
& +\operatorname{ord}_{p}\left(7 a+\lambda_{1} b\right)\left(7 a+\lambda_{2} b\right) .
\end{aligned}
$$

We continue with another two cases which are

$\min \left\{\operatorname{ord}_{p} U, \operatorname{ord}_{p} V\right\}=\operatorname{ord}_{p} U$ and

$\min \left\{\operatorname{ord}_{p} U, \operatorname{ord}_{p} V\right\}=\operatorname{ord}_{p} V$.

For both cases, we have

$$
\begin{aligned}
& \operatorname{ord}_{p}\left(Y+y_{0}\right) \geq \frac{1}{6} \min \left\{\operatorname{ord}_{p} r, \operatorname{ord}_{p} \lambda_{i} s\right\} \\
& -\frac{1}{2} \min \left\{\operatorname{ord}_{p} c m, \text { ord }_{p} k d\right\} \\
& -\min \left\{\operatorname{ord}_{p} a c, \operatorname{ord}_{p} b^{2}\right\} \\
& \quad-\frac{1}{3} \min \left\{\text { ord }_{p} d n, \text { ord }_{p} \text { em }\right\}
\end{aligned}
$$

where $i=1,2$.

By hypothesis, we substitute $\alpha$ and $\delta$, we have

$$
\operatorname{ord}_{p}\left(Y+y_{0}\right) \geq \frac{1}{6}(\alpha-22 \delta) .
$$

For Case (ii), equation (3) becomes

$$
\begin{aligned}
& \operatorname{ord}_{p}\left(Y+y_{0}\right) \\
& \geq \min \left\{\operatorname{ord}_{p} U, \operatorname{ord}_{p} V\right\}-\frac{1}{2} \operatorname{ord}_{p}(5 c m-k d) \\
& \quad+\frac{1}{2} \operatorname{ord}_{p}(21 d n-3 e m)-\operatorname{ord}_{p}\left(14 a c-6 b^{2}\right) \\
& \quad+\operatorname{ord}_{p}\left(7 a+\lambda_{1} b\right)\left(7 a+\lambda_{2} b\right) .
\end{aligned}
$$

It is same as (4). As a result, we will get (6). Now, we need to obtain the $p$-adic size of $\left(X+x_{0}\right)$. By Lemma 1, equation (1) becomes $\operatorname{ord}_{p}\left(X+x_{0}\right)$

$$
\begin{aligned}
\geq & \min \left\{\operatorname{ord}_{p} \alpha_{1} V, \operatorname{ord}_{p} \alpha_{2} U\right\}-\frac{1}{2} \operatorname{ord}_{p}\left[(35 c n-e k)^{2}\right. \\
& -4(21 d n-3 e m)(5 c m-k d)]+\operatorname{ord}_{p} \\
& (21 d n-3 e m)-\operatorname{ord}_{p}\left(14 a c-6 b^{2}\right)+\operatorname{ord}_{p} \\
& \left(7 a+\lambda_{1} b\right)+\operatorname{ord}_{p}\left(7 a+\lambda_{2} b\right) .
\end{aligned}
$$

Since $\operatorname{ord}_{p}(35 c n-e k)^{2} \neq \operatorname{ord}_{p} 4(21 d n-3 e m)$

$(5 \mathrm{~cm}-k d)$, we consider two cases.

Case (iii): $\operatorname{ord}_{p}(35 c n-e k)^{2}>\operatorname{ord}_{p} 4(21 d n-3 e m)(5 c m-$ $k d)$,

Case (iv): $\operatorname{ord}_{p}(35 c n-e k)^{2}<\operatorname{ord}_{p} 4(21 d n-3 e m)(5 c m-$ $k d)$.

For Case (iii), equation (7) becomes

$$
\begin{aligned}
\operatorname{ord}_{p}\left(X+x_{0}\right) \geq & \min \left\{\operatorname{ord}_{p} \alpha_{1} V, \operatorname{ord}_{p} \alpha_{2} U\right\}-\frac{1}{2} \operatorname{ord}_{p} \\
& (5 c m-k d)+\frac{1}{2} \operatorname{ord}_{p}(21 d n-3 e m) \\
& -\operatorname{ord}_{p}\left(14 a c-6 b^{2}\right)+\operatorname{ord}_{p} \\
& \left(7 a+\lambda_{1} b\right)\left(7 a+\lambda_{2} b\right) .
\end{aligned}
$$

We continue with another two cases which are $\min \left\{\operatorname{ord}_{p} \alpha_{1} V, \operatorname{ord}_{p} \alpha_{2} U\right\}=\operatorname{ord}_{p} \alpha_{1} V \quad$ and $\min \left\{\operatorname{ord}_{p} \alpha_{1} V, \operatorname{ord}_{p} \alpha_{2} U\right\}=\operatorname{ord}_{p} \alpha_{2} U$.

For both cases, we obtain

$\operatorname{ord}_{p}\left(X+x_{0}\right) \geq$ $\frac{1}{6} \min \left\{\operatorname{ord}_{p} r, \operatorname{ord}_{p} \lambda_{i} s\right\}-\frac{1}{2} \min \left\{\operatorname{ord}_{p} c m, \operatorname{ord}_{p} k d\right\}$

$-\min \left\{\operatorname{ord}_{p} a c, \operatorname{ord}_{p} b^{2}\right\}-\frac{4}{3} \min \left\{\operatorname{ord}_{p} d n, \operatorname{ord}_{p} e m\right\}$

where $i=1,2$.

By hypothesis, we obtain

$$
\operatorname{ord}_{p}\left(X+x_{0}\right) \geq \frac{1}{6}(\alpha-34 \delta) .
$$

For Case (iv), equation (7) becomes

$\operatorname{ord}_{p}\left(X+x_{0}\right) \geq$

$\min \left\{\operatorname{ord}_{p} \alpha_{1} V, \operatorname{ord}_{p} \alpha_{2} U\right\}-\frac{1}{2} \operatorname{ord}_{p}[4(21 d n-3 e m)$

$(5 c m-k d)]+\operatorname{ord}_{p}(21 d n-3 e m)-\operatorname{ord}_{p}$

$\left(14 a c-6 b^{2}\right)+\operatorname{ord}_{p}\left(7 a+\lambda_{1} b\right)+\operatorname{ord}_{p}\left(7 a+\lambda_{2} b\right)$.

That is,

$$
\begin{aligned}
\operatorname{ord}_{p}\left(X+x_{0}\right) \geq & \min \left\{\operatorname{ord}_{p} \alpha_{1} V, \operatorname{ord}_{p} \alpha_{2} U\right\}-\frac{1}{2} \operatorname{ord}_{p} \\
& +\operatorname{ord}_{p}(5 c m-k d)+\frac{1}{2} \operatorname{ord}_{p} \\
& (21 d n-3 e m)-\operatorname{ord}_{p}\left(14 a c-6 b^{2}\right) \\
& +\operatorname{ord}_{p}\left(7 a+\lambda_{1} b\right)\left(7 a+\lambda_{2} b\right) .
\end{aligned}
$$

It is same as (8). As a result, we will get (9).

In order to see the validity of our result and by Bezout's Theorem, we have $\alpha>(n-1)^{2} \delta$. Then, we have $\alpha>36 \delta$ in which $\alpha-36 \delta$ is the minimum value that we can get. In Lemma 2, we have $\alpha-34 \delta$ and $\alpha-22 \delta$ are greater than the minimum value. Thus, our lemma is valid.

Lemma 3 Let $p>7$ be a prime and $a, b, c, d, e$, $k, m, n, r, s$ in $Z_{p}$. Suppose $\left(X+x_{0}, Y+y_{0}\right)$ in $\Omega_{p}^{2}, \delta=$ $\max _{\operatorname{ord}} a, \operatorname{ord}_{p} b, \operatorname{ord}_{p} c, \operatorname{ord}_{p} d, \operatorname{ord}_{p} e$, $\left.\operatorname{ord}_{p} k, \operatorname{ord}_{p} m, \operatorname{ord}_{p} n\right\}$ and $\operatorname{ord}_{p} r, \operatorname{ord}_{p} s \geq \alpha>\delta$. 
If $\operatorname{ord}_{p} U=\frac{1}{6} \operatorname{ord}_{p}\left(\frac{r+\lambda_{1} s}{7 a+\lambda_{1} b}\right)$ and $\operatorname{ord}_{p} V=\frac{1}{6} \operatorname{ord}_{p}\left(\frac{r+\lambda_{2} s}{7 a+\lambda_{2} b}\right)$ with the condition $\operatorname{ord}_{p}(35 c n-e k)^{2}=\operatorname{ord}_{p} 4(21 d n-$ $3 e m)(5 \mathrm{~cm}-k d)$ where $U=x+\alpha_{1} y$ and $V=x+\alpha_{2} y$, then $\operatorname{ord}_{p}\left(X+x_{0}\right) \geq \frac{1}{6}(\alpha-34 \delta)-\frac{1}{2} \omega_{0} \quad$ and $\quad \operatorname{ord}_{p}\left(Y+y_{0}\right) \geq$ $\frac{1}{6}(\alpha-22 \delta)-\frac{1}{2} \omega_{0}$ for some $\omega_{0} \geq 0$.

Proof. From Lemma 2, we have

$\operatorname{ord}_{p}\left(Y+y_{0}\right)$

$\geq \min \left\{\operatorname{ord}_{p} U, \operatorname{ord}_{p} V\right\}-\frac{1}{2} \operatorname{ord}_{p}\left[(35 c n-e k)^{2}\right.$

$-4(21 d n-3 e m)(5 c m-k d)]+\operatorname{ord}_{p}(21 d n-$

$3 e m)-\operatorname{ord}_{p}\left(14 a c-6 b^{2}\right)+\operatorname{ord}_{p}\left(7 a+\lambda_{1} b\right)$

$+\operatorname{ord}_{p}\left(7 a+\lambda_{2} b\right)$.

If $\min \left\{\operatorname{ord}_{p} U, \operatorname{ord}_{p} V\right\}=\operatorname{ord}_{p} U$, then we obtain

$\operatorname{ord}_{p}\left(Y+y_{0}\right) \geq$

$\frac{1}{6} \operatorname{ord}_{p}\left(\frac{r+\lambda_{1} s}{7 a+\lambda_{1} b}\right)-\frac{1}{2} \operatorname{ord}_{p}\left[(35 c n-e k)^{2}-4(21 d n\right.$

$-3 e m)(5 c m-k d)]+\operatorname{ord}_{p}(21 d n-3 e m)-\operatorname{ord}_{p}$

$\left(14 a c-6 b^{2}\right)+\operatorname{ord}_{p}\left(7 a+\lambda_{1} b\right)+\operatorname{ord}_{p}\left(7 a+\lambda_{2} b\right)$.

Now, let $\operatorname{ord}_{p}(35 c n-e k)^{2}=\operatorname{ord}_{p} 4(21 d n-3 e m)(5 c m-$ $k d)=\gamma$, we have $(35 c n-e k)^{2}=A p^{\gamma}$ and $4(21 d n-$ $3 e m)(5 c m-k d)=B p^{\gamma}$ where $\operatorname{ord}_{p} A=\operatorname{ord}_{p} B=0$. Then, $\operatorname{ord}_{p}\left[(35 c n-e k)^{2}-4(21 d n-3 e m)(5 c m-k d)\right]$

$=\operatorname{ord}_{p}\left(A p^{\gamma}-B p^{\gamma}\right)=\gamma+\omega_{0}$

where $\omega_{0}=\operatorname{ord}_{p}(A-B) \geq 0$.

Now, we choose $\gamma=\operatorname{ord}_{p} 4(21 \mathrm{dn}-3 \mathrm{em})(5 \mathrm{~cm}-k d)$ and substitute the expression of $\lambda_{1}, \lambda_{2}$. Then,

$\operatorname{ord}_{p}\left(Y+y_{0}\right) \geq$

$\frac{1}{6} \operatorname{ord}_{p}\left(r+\lambda_{1} s\right)-\frac{1}{2} \operatorname{ord}_{p}(5 c m-k d)-\frac{1}{3} \operatorname{ord}_{p}$

$(21 d n-3 e m)-\operatorname{ord}_{p}\left(14 a c-6 b^{2}\right)-\frac{1}{2} \omega_{0}$.

By using the hypothesis, we have

$$
\operatorname{ord}_{p}\left(Y+y_{0}\right) \geq \frac{1}{6}(\alpha-22 \delta)-\frac{1}{2} \omega_{0}
$$

for some $\omega_{0} \geq 0$.

If $\min \left\{\operatorname{ord}_{p} U, \operatorname{ord}_{p} V\right\}=\operatorname{ord}_{p} V$, then we obtain

$\operatorname{ord}_{p}\left(Y+y_{0}\right) \geq$

$\frac{1}{6} \operatorname{ord}_{p}\left(\frac{r+\lambda_{2} s}{7 a+\lambda_{2} b}\right)-\frac{1}{2} \operatorname{ord}_{p}\left[(35 c n-e k)^{2}\right.$

$-4(21 d n-3 e m)(5 c m-k d)]+\operatorname{ord}_{p}(21 d n$

$-3 e m)-\operatorname{ord}_{p}\left(14 a c-6 b^{2}\right)+\operatorname{ord}_{p}\left(7 a+\lambda_{1} b\right)$

$+\operatorname{ord}_{p}\left(7 a+\lambda_{2} b\right)$.

By substituting the expression of $\lambda_{1}, \lambda_{2}$ and using the hypothesis, we have

$$
\operatorname{ord}_{p}\left(Y+y_{0}\right) \geq \frac{1}{6}(\alpha-22 \delta)-\frac{1}{2} \omega_{0}
$$

for some $\omega_{0} \geq 0$.

Also, from Lemma 2, we have

$\operatorname{ord}_{p}\left(X+x_{0}\right) \geq$

$\min \left\{\operatorname{ord}_{p} \alpha_{1} V, \operatorname{ord}_{p} \alpha_{2} U\right\}-\frac{1}{2} \operatorname{ord}_{p}\left[(35 c n-e k)^{2}\right.$

$-4(21 d n-3 e m)(5 c m-k d)]+\operatorname{ord}_{p}(21 d n$

$-3 e m)-\operatorname{ord}_{p}\left(14 a c-6 b^{2}\right)+\operatorname{ord}_{p}\left(7 a+\lambda_{1} b\right)$

$+\operatorname{ord}_{p}\left(7 a+\lambda_{2} b\right)$.

If $\min \left\{\operatorname{ord}_{p} \alpha_{1} V, \operatorname{ord}_{p} \alpha_{2} U\right\}=\operatorname{ord}_{p} \alpha_{1} V$. By using the same argument, we obtain

$$
\begin{aligned}
\operatorname{ord}_{p}\left(X+x_{0}\right) \geq \frac{1}{6} & \operatorname{ord}_{p}\left(r+\lambda_{2} s\right)-\frac{1}{2} \operatorname{ord}_{p}(5 c m-k d) \\
& -\frac{4}{3} \operatorname{ord}_{p}(21 d n-3 e m) \\
& \quad-\operatorname{ord}_{p}\left(14 a c-6 b^{2}\right)-\frac{1}{2} \omega_{0} .
\end{aligned}
$$

For the case $\min \left\{\operatorname{ord}_{p} \alpha_{1} V, \operatorname{ord}_{p} \alpha_{2} U\right\}=\operatorname{ord}_{p} \alpha_{2} U$. By using the similar manner, we have

$$
\begin{aligned}
\operatorname{ord}_{p}\left(X+x_{0}\right) \geq \frac{1}{6} & \operatorname{ord}_{p}\left(r+\lambda_{1} s\right)-\frac{1}{2} \operatorname{ord}_{p}(5 c m-k d) \\
- & \frac{4}{3} \operatorname{ord}_{p}(21 d n-3 e m) \\
& \quad-\operatorname{ord}_{p}\left(14 a c-6 b^{2}\right)-\frac{1}{2} \omega_{0} .
\end{aligned}
$$

By hypothesis, we have the following result:

$$
\operatorname{ord}_{p}\left(X+x_{0}\right) \geq \frac{1}{6}(\alpha-34 \delta)-\frac{1}{2} \omega_{0}
$$

for some $\omega_{0} \geq 0$ as asserted.

Now, we will prove the Theorem 2.

Proof of Theorem 2.

Let $g=f_{x}$ and $h=f_{y}$. Suppose $x=X+x_{0}$ and $y=Y+y_{0}$. By completing the sixth degree,

$$
\begin{aligned}
\frac{g+\lambda h}{7 a+\lambda b}=\left[\left(X+x_{0}\right)+\right. & \left.\frac{1}{6}\left(\frac{6 b+2 \lambda c}{7 a+\lambda b}\right)\left(Y+y_{0}\right)\right]^{6} \\
& +\left(\frac{r+\lambda s}{7 a+\lambda b}\right)
\end{aligned}
$$

with

$$
\begin{aligned}
& \left(\frac{6 b+2 \lambda c}{7 a+\lambda b}\right)^{2}-\frac{36}{15}\left(\frac{5 c+3 \lambda d}{7 a+\lambda b}\right)=0 \\
& \left(\frac{6 b+2 \lambda c}{7 a+\lambda b}\right)^{3}-\frac{54}{5}\left(\frac{4 d+4 \lambda e}{7 a+\lambda b}\right)=0 \\
& \left(\frac{6 b+2 \lambda c}{7 a+\lambda b}\right)^{4}-\frac{432}{5}\left(\frac{3 e+5 \lambda k}{7 a+\lambda b}\right)=0 \\
& \left(\frac{6 b+2 \lambda c}{7 a+\lambda b}\right)^{5}-6^{4}\left(\frac{2 k+6 \lambda m}{7 a+\lambda b}\right)=0 \\
& \left(\frac{6 b+2 \lambda c}{7 a+\lambda b}\right)^{6}-6^{6}\left(\frac{m+7 \lambda n}{7 a+\lambda b}\right)=0
\end{aligned}
$$

By solving (11), (12), (13), (14) and (15) simultaneously, we obtain a quadratic equation

$$
(21 d n-3 e m) \lambda^{2}+(35 c n-e k) \lambda+(5 c m-k d)=0 .
$$


Let

$$
\begin{aligned}
& U=\left(X+x_{0}\right)+\frac{1}{6}\left(\frac{6 b+2 \lambda_{1} c}{7 a+\lambda_{1} b}\right)\left(Y+y_{0}\right) \\
& V=\left(X+x_{0}\right)+\frac{1}{6}\left(\frac{6 b+2 \lambda_{2} c}{7 a+\lambda_{2} b}\right)\left(Y+y_{0}\right),
\end{aligned}
$$

we have

$$
\begin{aligned}
& g+\lambda_{1} h=\left(7 a+\lambda_{1} b\right) U^{6}+r+\lambda_{1} s \\
& g+\lambda_{2} h=\left(7 a+\lambda_{2} b\right) V^{6}+r+\lambda_{2} s .
\end{aligned}
$$

We let $F(U, V)=g+\lambda_{1} h$ and $G(U, V)=g+\lambda_{2} h$.

The combination of the indicator diagrams associated with the Newton polyhedron of (18) and (19) as shown in Figure 1. There exists a point $(U, V)$ such that $F(U, V)=0$ and $G(U, V)=0$ where $\left(\mu_{1}, \mu_{2}\right)$ is the point of intersection in the indicator diagrams of $F(U, V)$ and $G(U, V)$.

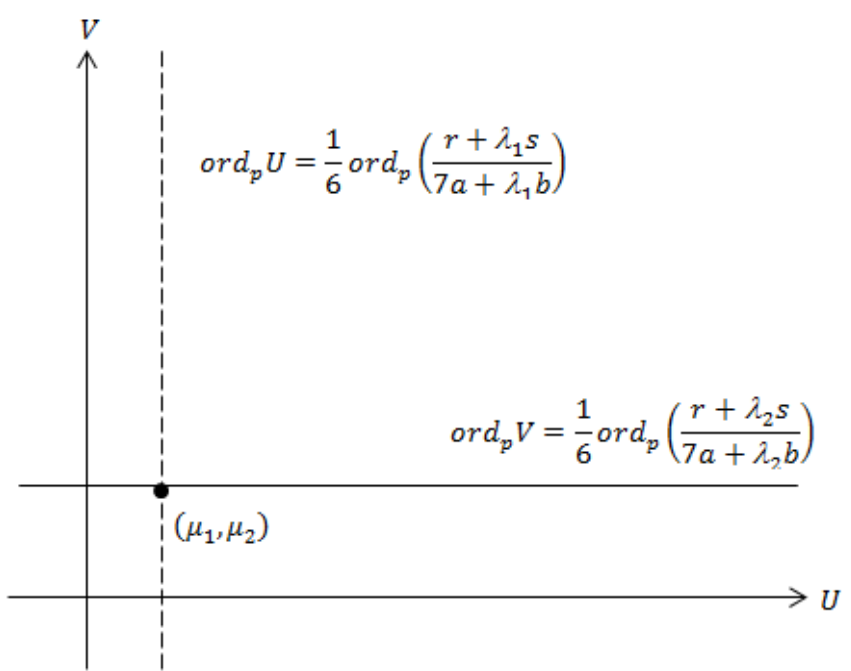

Figure 1. The indicator diagrams for the polynomials of $F(U, V)$ (dash line) and $G(U, V)$ (solid line).

Let $U=\widehat{U}$ and $V=\widehat{V}$. From (16) and (17), there exists $(\widehat{X}+$ $\left.\hat{x}_{o}\right)$ and $\left(\hat{Y}+\hat{y}_{o}\right)$ in such a way that:

$$
\left(\hat{X}+\hat{x}_{0}\right)=\frac{\alpha_{1} \widehat{V}-\alpha_{2} \widehat{U}}{\alpha_{1}-\alpha_{2}},\left(\hat{Y}+\hat{y}_{0}\right)=\frac{\widehat{U}-\widehat{V}}{\alpha_{1}-\alpha_{2}}
$$

where $\alpha_{1}=\frac{6 b+2 \lambda_{1} c}{6\left(7 a+\lambda_{1} b\right)}, \alpha_{2}=\frac{6 b+2 \lambda_{2} c}{6\left(7 a+\lambda_{2} b\right)}$ and $\lambda_{1}, \lambda_{2}$ are the zeros of $z(\lambda)$ in Lemma 1. Next, we find $\operatorname{ord}_{p} \hat{X}$ and $\operatorname{ord}_{p} \hat{Y}$. From Lemma 2, we have

$$
\begin{aligned}
& \operatorname{ord}_{p}\left(\hat{X}+\hat{x}_{0}\right) \geq \frac{1}{6}(\alpha-34 \delta), \\
& \operatorname{ord}_{p}\left(\hat{Y}+\hat{y}_{0}\right) \geq \frac{1}{6}(\alpha-22 \delta) .
\end{aligned}
$$

By the following property,

$\operatorname{ord}_{p}(A \pm B) \geq \min \left\{\operatorname{ord}_{p} A, \operatorname{ord}_{p} B\right\}$, we have

$$
\begin{aligned}
& \operatorname{ord}_{p}\left(\hat{X}+\hat{x}_{0}\right) \geq \operatorname{ord}_{p} \hat{X}+\varepsilon_{1} \\
& \operatorname{ord}_{p}\left(\hat{Y}+\hat{y}_{0}\right) \geq \operatorname{ord}_{p} \hat{Y}+\varepsilon_{2}
\end{aligned}
$$

for some $\varepsilon_{1}, \varepsilon_{2} \geq 0$. Thus, we will have

$$
\begin{aligned}
& \operatorname{ord}_{p} \hat{X} \geq \frac{1}{6}(\alpha-34 \delta)-\varepsilon_{1}, \\
& \operatorname{ord}_{p} \hat{Y} \geq \frac{1}{6}(\alpha-22 \delta)-\varepsilon_{2} .
\end{aligned}
$$

We let $\xi=\hat{X}+\hat{x}_{o}$ and $\eta=\hat{Y}+\hat{y}_{o}$, then

$$
\begin{aligned}
& \operatorname{ord}_{p}\left(\xi-\hat{x}_{o}\right) \geq \frac{1}{6}(\alpha-34 \delta)-\varepsilon_{1}, \\
& \operatorname{ord}_{p}\left(\eta-\hat{y}_{o}\right) \geq \frac{1}{6}(\alpha-22 \delta)-\varepsilon_{2} .
\end{aligned}
$$

By back substitution, we have

$g(\xi, \eta)=f_{x}(\xi, \eta)=o$ and $h(\xi, \eta)=f_{y}(\xi, \eta)=o$.

From Lemma 3, we have

$$
\begin{aligned}
& \operatorname{ord}_{p}\left(\hat{X}+\hat{x}_{o}\right) \geq \frac{1}{6}(\alpha-34 \delta)-\frac{1}{2} \omega_{o} \\
& \operatorname{ord}_{p}\left(\hat{Y}+\hat{y}_{o}\right) \geq \frac{1}{6}(\alpha-22 \delta)-\frac{1}{2} \omega_{o} .
\end{aligned}
$$

By the same property, we have

$$
\begin{aligned}
& \operatorname{ord}_{p}\left(\hat{X}+\hat{x}_{0}\right) \geq \operatorname{ord}_{p} \hat{X}+\varepsilon_{3} \\
& \operatorname{ord}_{p}\left(\hat{Y}+\hat{y}_{0}\right) \geq \operatorname{ord}_{p} \hat{Y}+\varepsilon_{4}
\end{aligned}
$$

for some $\varepsilon_{3}, \varepsilon_{4} \geq 0$. Thus, we will obtain

$$
\begin{aligned}
& \operatorname{ord}_{p} \hat{X} \geq \frac{1}{6}(\alpha-34 \delta)-\varepsilon_{3}-\frac{1}{2} \omega_{0}, \\
& \operatorname{ord}_{p} \hat{Y} \geq \frac{1}{6}(\alpha-22 \delta)-\varepsilon_{4}-\frac{1}{2} \omega_{0} .
\end{aligned}
$$

We let $\xi=\hat{X}+\hat{x}_{o}$ and $\eta=\hat{Y}+\hat{y}_{o}$, then

$$
\begin{aligned}
& \operatorname{ord}_{p}\left(\xi-\hat{x}_{o}\right) \geq \frac{1}{6}(\alpha-34 \delta)-\varepsilon_{3}-\frac{1}{2} \omega_{0}, \\
& \operatorname{ord}_{p}\left(\eta-\hat{y}_{0}\right) \geq \frac{1}{6}(\alpha-22 \delta)-\varepsilon_{4}-\frac{1}{2} \omega_{0} .
\end{aligned}
$$

By back substitution, we have

$$
g(\xi, \eta)=f_{x}(\xi, \eta)=0 \text { and } h(\xi, \eta)=f_{y}(\xi, \eta)=0
$$

\section{ESTIMATION OF CARDINALITY}

$$
N\left(f_{x}, f_{y} ; p^{\alpha}\right)
$$

From Loxton \& Smith (1982), we can get the $N\left(f_{x}, f_{y} ; p^{\alpha}\right)$ from the $p$-adic size of $\operatorname{ord}_{p}\left(x-\xi_{i}\right)$ and $\operatorname{ord}_{p}\left(y-\eta_{i}\right)$ by the following theorem.

Theorem 3 Let $p$ be a prime and $g(x, y)$ and $h(x, y)$ are polynomials in $Q_{p}[x, y]$. Let $\alpha>0,\left(\xi_{i}, \eta_{i}\right), i \geq 0$ be common zeros of $g$ and $h$, and $\gamma_{i}(\alpha)=\inf _{x \in H(\alpha)}\left\{\operatorname{ord}_{p}(x-\right.$ $\left.\left.\xi_{i}\right), \operatorname{ord}_{p}\left(y-\eta_{i}\right)\right\}$ where $H(\alpha)=\cup_{i} H_{i}(\alpha)$. If $\alpha>\gamma_{i}(\alpha)$, then $N\left(g, h ; p^{\alpha}\right) \leq \sum_{i} p^{2\left(\alpha-\gamma_{i}(\alpha)\right)}$

Next, we can prove the following theorem.

Theorem 4 Let $f(x, y)=a x^{7}+b x^{6} y+c x^{5} y^{2}+d x^{4} y^{3}+$ $e x^{3} y^{4}+k x^{2} y^{5}+m x y^{6}+n y^{7}+r x+s y+t$ be a polynomial in $Z_{p}[x, y]$ with $p>7$ is a prime. Let $\alpha>0, \delta=$ $\max \left\{\operatorname{ord}_{p} a, \operatorname{ord}_{p} b, \operatorname{ord}_{p} c\right.$, 
$\left.\operatorname{ord}_{p} d, \operatorname{ord}_{p} e, \operatorname{ord}_{p} k, \operatorname{ord}_{p} m, \operatorname{ord}_{p} n\right\}$, then

$$
N\left(f_{x}, f_{y} ; p^{\alpha}\right) \leq \begin{cases}p^{2 \alpha} & \text { if } \alpha \leq \delta \\ 36 p^{68 \delta+12 q} & \text { if } \alpha>\delta\end{cases}
$$

where $q=\max \left\{\varepsilon_{1}, \varepsilon_{3}+\frac{1}{2} \omega_{0}\right\}$.

Proof. If $\alpha \leq \delta$, then $N\left(f_{x}, f_{y} ; p^{\alpha}\right) \leq p^{2 \alpha}$ since $\gamma_{i}(\alpha)=0$. If $\alpha>\delta$, from Theorem 3, we have

$$
\operatorname{ord}_{p}\left(\xi-x_{0}\right) \geq \frac{1}{6}(\alpha-34 \delta)-q
$$

where $q=\max \left\{\varepsilon_{1}, \varepsilon_{3}+\frac{1}{2} \omega_{0}\right\}$. We obtain

$$
\alpha-6 \gamma_{i}(\alpha) \leq 34 \delta+6 q \text {. }
$$

From Bezout's Theorem, the product of the degrees of $f_{x}$ and $f_{y}$ is the maximum number of the common zeros. Therefore,

$$
N\left(f_{x}, f_{y} ; p^{\alpha}\right) \leq 36 p^{68 \delta+12 q}
$$

for $\alpha>\delta$ and $q=\max \left\{\varepsilon_{1}, \varepsilon_{3}+\frac{1}{2} \omega_{0}\right\}$.

\section{ESTIMATION OF EXPONENTIAL SUMS $\boldsymbol{S}\left(\boldsymbol{f} ; p^{\alpha}\right)$}

The exponential sums can be estimated by using the theorems in Mohd. Atan (1984).

Theorem 5 Let $p$ be a prime and $f(x, y)$ be a polynomial in $Z_{p}[x, y]$. For $\alpha>1, \theta=\frac{\alpha}{2}$, let

$$
S\left(f ; p^{\alpha}\right)=\sum_{x, y \bmod p} e^{\frac{2 \pi i f(x, y)}{p^{\alpha}}} .
$$

Then, $\quad\left|S\left(f ; p^{\alpha}\right)\right| \leq p^{2(\alpha-\theta)} N_{f_{x} f_{y}}\left(p^{\theta}\right)$.

If $\alpha$ is odd, then we use the next theorem.

Theorem 6 Let $p$ be a prime and $f(x, y)$ be a polynomial in $Z_{p}[x, y]$. Let $\alpha=2 \beta+1$, where $\beta \geq 1$ and

$$
S\left(f ; p^{\alpha}\right)=\sum_{x, y \bmod p} e^{\frac{2 \pi i f(x, y)}{p^{\alpha}}},
$$

then

$$
\left|S\left(f ; p^{\alpha}\right)\right| \leq p^{2 \beta+2} N_{f_{x} f_{y}}\left(p^{\beta}\right) .
$$

By using the above two theorems, we have the following result.

Theorem 7 Let $f(x, y)=a x^{7}+b x^{6} y+c x^{5} y^{2}+d x^{4} y^{3}+$ $e x^{3} y^{4}+k x^{2} y^{5}+m x y^{6}+n y^{7}+r x+s y+t$ be a polynomial in $Z_{p}[x, y]$. Suppose $p>7$ is a prime and $\alpha>1$. Let $\delta=$ $\max \left\{\operatorname{ord}_{p} a, \operatorname{ord}_{p} b\right.$, $\left.\operatorname{ord}_{p} C, \operatorname{ord}_{p} d, \operatorname{ord}_{p} e, \operatorname{ord}_{p} k, \operatorname{ord}_{p} m, \operatorname{ord}_{p} n\right\}$, then

$$
\left|S\left(f ; p^{\alpha}\right)\right| \leq \min \left\{p^{2 \alpha}, 36 p^{\alpha+1+68 \delta+12 q}\right\}
$$

where $q=\max \left\{\varepsilon_{1}, \varepsilon_{3}+\frac{1}{2} \omega_{0}\right\}$.

Proof. From Theorem 4, we have

$$
N\left(f_{x}, f_{y} ; p^{\alpha}\right) \leq \min \left\{p^{2 \alpha}, 36 p^{68 \delta+12 q}\right\}
$$

where $\theta=\frac{\alpha}{2}$ and $q=\max \left\{\varepsilon_{1}, \varepsilon_{3}+\frac{1}{2} \omega_{0}\right\}$.

Suppose $\alpha$ is even. If $\alpha>1$ and $\alpha=2 \theta$. By using Theorem 5, we have

$$
\left|S\left(f ; p^{\alpha}\right)\right| \leq \min \left\{p^{2 \alpha}, 36 p^{\alpha+68 \delta+12 q}\right\} .
$$

Suppose $\alpha$ is odd. If $\alpha>1$ and $\alpha=2 \beta+1$. By using Theorem 6, we have

$$
\left|S\left(f ; p^{\alpha}\right)\right| \leq \min \left\{p^{2 \alpha}, 36 p^{\alpha+1+68 \delta+12 q}\right\} .
$$

\section{CONCLUSION}

The exponential sums of the seventh-degree polynomial with two variables in the form

$$
\begin{gathered}
f(x, y)=a x^{7}+b x^{6} y+c x^{5} y^{2}+d x^{4} y^{3}+e x^{3} y^{4} \\
+k x^{2} y^{5}+m x y^{6}+n y^{7}+r x+s y+t
\end{gathered}
$$

in $Z_{p}[x, y]$ is given by

$$
\left|S\left(f ; p^{\alpha}\right)\right| \leq \min \left\{p^{2 \alpha}, 36 p^{\alpha+1+68 \delta+12 q}\right\}
$$

where $p, q, \alpha$ and $\delta$ are defined in Theorem 7 .

\section{ACKNOWLEDGEMENT}

We take this opportunity to express our gratitude for the financial support from Graduate Research Fellowship of UPM, grant UPM/700-2/1/GBP/2017/9597900 and those helps in this research to make it success. 


\section{REFERENCES}

Aminudin, S. S., Sapar, S. H. \& Mohd. Atan, K. A. (2014). Mohd. Atan, K. A. (1984). Newton polyhedral and estimates for method of estimating the p-adic sizes of common zeros of exponential sums. Ph.D. Thesis, University of New partial derivative polynomials associated with a complete South Wales, Kensington, Australia. cubic form. International Conference on Mathematicßohd. Atan, K. A. \& Loxton, J. H. (1986). Newton Sciences and Statistics 2013, 205-212. Polyhedra and Solutions of Congruences. In

Chan, K. L. \& Mohd. Atan, K. A. (1997). On the estimate to Proceeding of Diophantine Analysis, Cambridge solutions of congruence equations associated with a University Press, 67-82. quartic form. J. Phys. Sci., 8, 21-34.

Mohd. Atan, K. A. (1986). Newton polyhedral method of

Heng, S. H. \& Mohd. Atan, K. A. (1999). An estimation of determining p-adic orders of zeros common to two exponential sums associated with a cubic form. J. Phys. Sci., polynomials in $Q_{p}[x, y]$. Pertanika, 9(3), 375-380. 10, 1-21.

Koblitz, N. (1977). p-adic Numbers, p-adic Analysis and Zeta-Functions. New York, Second Edition (SpringerVerlag), 89-99.

Lasaraiya, S., Sapar, S. H. \& Johari, M. A. M. (2016a). cardinality of the twelfth-degree polynomial. In Conference Proceedings, 020008, AIP Publishing.
Sapar, S. H. \& Mohd. Atan, K. A. (2002). Estimate for the cardinality of the set of solution to congruence equations. J. Technology, 36(C), 13-40.

Sapar, S. H. \& Mohd. Atan, K. A. (2009). A method of On the estimating the p-adic sizes of common zeros of partial AIP derivative polynomials associated with a quintic form. World Scientific, 5, 541-554.

Lasaraiya, S., Sapar, S. H. \& Johari, M. A. M. (2016b). On tsapar, S. H., Mohd. Atan, K. A. \& Aminuddin, S. H. (2013). An cardinality of the set of solutions to congruence equation estimating the p-adic sizes of common zeros of partial associated with polynomial of degree eleven. In P Conference derivative polynomials. New Trends in Mathematical Sciences, Proceedings, 050015, AIP Publishing.

Loxton, J. H. \& Smith, R. A. (1982). Estimate for multiple exponential sums. J. Aust. Math. Soc., 33, 125-134.

Loxton, J. H. \& Vaughan, R. C. (1985). The estimate of complete exponential sums. Canad. Math. Bull., 28(4), 440-454. 1(1), 38-48.

Sapar, S. H., Aminudin, S. S. \& Mohd. Atan, K. A. (2014). A method of estimating the p-adic sizes polynomial. International Journal of Pure Mathematics, 1, 22-29.

Yap, H. K., Sapar, S. H. \& Mohd. Atan, K. A. (2011). Estimation of p-adic sizes of common zeros of partial derivative associated with a cubic form. Sains Malaysiana, 40(8), 921-926. 